Mots. Les langages du politique

112 | 2016

Discours présidentiels et de présidentielles

\title{
Discours présidentiels au Portugal. Un regard pluridisciplinaire
}

Portuguese presidential speeches. A multidisciplinary perspective

Discursos presidenciales en Portugal. Un abordaje multidisciplinar

Maria-Aldina Marques

(2) OpenEdition

Journals

Édition électronique

URL : https://journals.openedition.org/mots/22534

DOI : $10.4000 /$ mots. 22534

ISSN : 1960-6001

Éditeur

ENS Éditions

Édition imprimée

Date de publication : 15 octobre 2016

Pagination : 125-132

ISBN : 978-2-84788-850-8

ISSN : 0243-6450

Référence électronique

Maria-Aldina Marques, « Discours présidentiels au Portugal. Un regard pluridisciplinaire », Mots. Les

langages du politique [En ligne], 112 | 2016, mis en ligne le 15 octobre 2018, consulté le 22 avril 2022

URL : http://journals.openedition.org/mots/22534 ; DOI : https://doi.org/10.4000/mots.22534

(c) ENS Éditions 


\section{Discours présidentiels au Portugal. Un regard pluridisciplinaire}

La République portugaise fut instaurée le 5 octobre 1910, après un coup d’État qui mit fin à huit siècles de régime monarchique. Le premier président élu fut Manuel de Arriaga (1911-1915), et le dernier, avant le renouvellement de 2016, Cavaco Silva, dix-neuvième président de la République portugaise ${ }^{1}$. Dix-neuf personnalités différentes ont donc siégé au Palais de Belém (la résidence officielle du président de la République portugaise) avant l'actuelle présidence, au cours de trois périodes historiques : la Première République (1910-1926), une période instable de quinze ans ; la Deuxième République (1926-1974), une longue période de quarante-huit ans de dictature, après un coup d’État militaire en 1926 qui donna lieu, en 1933, au régime totalitaire de Salazar, le Estado Novo [État Nouveau] ; et la Troisième République, à partir de 1974, avec la Révolution des Fillets, jusqu'à aujourd'hui. Le mandat le plus court fut celui de Mendes Cabeçadas (dix-sept jours, du 31 mai au 17 juin 1926) et le plus long celui d'Óscar Carmona (presque vingt-cinq ans, du 16 novembre 1926 jusqu'à sa mort, survenue le 18 avril 1951). La Troisième République est marquée par une homogénéité temporelle, tous les présidents ayant été élus pour deux quinquennats (les deux mandats successifs prévus par la Constitution portugaise) ${ }^{2}$.

Les études sur cette institution républicaine, la présidence de la République, notamment en ce qui concerne les discours présidentiels, sont récentes. En fait, la période de la dictature a été peu propice à l'étude des discours politiques; il faut d'ailleurs souligner que la dictature est la période d'un « dictateur fort et un président faible», marquée par la «discrétion et l'absence d'une image [de président] manifestement politique» (Freire, Pinto, 2005, p. 31 et 33)3. Par

1. Après les élections du 24 janvier 2016, le nouveau président de la République portugaise est Marcelo Rebelo de Sousa.

2. Le président de la République portugaise est élu pour cinq ans au suffrage universel direct.

3. La subordination du président face au tout-puissant chef du gouvernement est une constante de la dictature, à laquelle seulement Craveiro Lopes a réagi. L'affrontement entre celui-ci (président de la République de 1951 à 1958) et le dictateur Salazar est présenté aussi en termes de dévalorisation des discours. La parole du président a été censurée : «Les discours étaient modifiés, les projets rejetés... » (site web de la présidence de la République portugaise). Pour rendre mon texte plus accessible, j'ai traduit toutes les citations portugaises en français.

Universidade do Minho (Portugal), CEHUM

mamarques@ilch.uminho.pt 
conséquent, le discours politique en général, et le discours présidentiel en particulier, ne sont objets d'analyse pour les sciences humaines et sociales qu'après la Révolution de 1974, et c'est la Troisième République qui suscite la plupart de ces recherches.

En tant qu'objets de recherche, les discours présidentiels sont étudiés par des disciplines appartenant à plusieurs domaines scientifiques, notamment les sciences du langage, les sciences politiques et les sciences de la communication.

Nous nous attacherons à présenter ces approches diversifiées des discours présidentiels au Portugal, et en particulier les genres de discours privilégiés, les méthodologies utilisées et les résultats de ces recherches, en les considérant d'un point de vue pluridisciplinaire4.

\section{Le statut de président de la République portugaise}

La caractérisation du régime politique portugais n'est pas aisée : «semiprésidentiel » (Freire, Pinto, 2005; Espírito Santo, 2007), «mixte », « parlementaire », sont des catégorisations issues d'une controverse autour de ce sujet, présentée et longuement discutée par Rapaz qui, à son tour, parle de «l'erreur semi-présidentielle» (2012, p. 446).

Malgré ce point de départ, les auteurs s'accordent à considérer que le président de la République a un pouvoir qui se veut modérateur et unificateur de la nation - on attend du président qu'il soit le garant de la Constitution - et autonome face aux pouvoirs exécutif et législatif : "Il est visible que les constituants originaires et dérivés ont voulu extraire le président de la République du monde partisan et du pouvoir exécutif »(Rapaz, 2008). De plus, cette autonomie est affirmée notamment par une prise de position supra-partisane, pouvant être symboliquement marquée par la suspension de l'affiliation du candidat à son parti politique (Freire, Pinto, 2005, p. 86).

Néanmoins, cette autonomisation présidentielle est du domaine de l'éthique politique plus qu'elle n'est une pratique politique effective, étant donné le soutien apporté par les partis politiques aux candidats présidentiels5. D’où le symbolisme accordé à l'énoncé : «Je suis le président de tous les Portugais ».

Cette déclaration, prononcée d'abord par le premier président de la période démocratique actuelle, pendant une campagne électorale, a été reprise par Mário Soares dans son discours de victoire, en 1996, et est devenue «canonique».

4. Je reprends Charaudeau (2010, p.4) : «Je considère que la pluridisciplinarité est "une addition de disciplines, sans véritable interaction entre elles" [...], ce qui fait que chaque discipline garde son autonomie, ne réinterroge pas ses présupposés au regard de l'autre discipline, et ne fait qu'apporter [...] son propre éclairage sur un objet d'étude lui-même analysé par d'autres. »

5. D’où le besoin éprouvé par Mário Soares, et repris par les autres candidats, de proclamer l'extinction de la majorité qui l'avait soutenu, une majorité populaire, certes, mais aussi partisane. 
Le fait que l'élection présidentielle soit la seule à être unipersonnelle, en dehors des relations de forces partisanes, met en relief la figure présidentielle.

\section{Le président / les présidentielles dans les discours}

On constate un positionnement «interne» de valorisation des discours présidentiels. En fait, les présidents, et les candidats à la présidence de la République, sont conscients de l'importance des discours. Manuel Alegre, candidat à l'élection présidentielle de 2006 , le reconnaît et l'affirme dans son Manifeste électoral : "Le mot est l'arme la plus importante d'un président. Les mots changent la vie, ils aident à bâtir la confiance et l'espoir» (voir Pinto, 2012, p. 200).

On pourrait penser que ce n'est qu'une figure de style de la part de ce candidat, un poète dans ce cas précis, et donc sensible aux «mots », mais il s'agit d'un positionnement récurrent qui dénote l'attention portée aux discours (aux mots) par les candidats/présidents eux-mêmes. Notons que les derniers présidents portugais (des présidents civils, après soixante ans de présidents militaires) ont un sens aigu de l'importance de leurs discours, qu'ils ont fait publier au fur et à mesure de leurs mandats présidentiels. Entre 1986 et 1996, les deux quinquennats de sa présidence, Mário Soares a publié dix volumes, intitulés Intervenções (Interventions); Jorge Sampaio, président de 1996 à 2006, a publié neuf volumes, intitulés Portugueses (Portugais), entre 1997 et 2007; et finalement, Cavaco Silva, à la présidence dès 2006, a publié entre 2007 et 2015 neuf volumes, les Roteiros (Feuilles de route).

La Troisième République est la période qui concentre l'attention de la communauté scientifique, même si toute la période républicaine est étudiée (Freire, Pinto, 2005; Pinto, 2001).

En mettant l'accent sur l'objet de recherche, on voit que l'intérêt pour les présidents et les discours présidentiels mobilise différentes disciplines des sciences sociales et humaines, présentant des approches qui se croisent sans qu'il y ait, pour autant, des travaux conjoints : elles gardent leur autonomie et, globalement, ignorent les acquis des autres. Un examen même superficiel des références bibliographiques des travaux publiés suffit pour se rendre compte de cet isolement disciplinaire.

Trois perspectives ressortent quand on considère l'institution et les discours présidentiels.

D'abord, le groupe des disciplines telles que les sciences juridiques, la sociologie politique ou les sciences politiques (Sousa, 1998), probablement le groupe le plus nombreux, et le plus influent aussi, dont l'objectif est d' «éclairer la place du président de la République au sein des institutions politiques portugaises" (Rapaz, 2013, p.3). Ces réflexions sont axées sur des thèmes 
centraux tels que les caractéristiques du régime démocratique portugais et la pratique du pouvoir par les présidents ou, en d'autres termes, leur légitimité.

L'intérêt porté à l'institution présidentielle s'articule ainsi aux catégorisations du régime démocratique portugais et aux caractéristiques de la présidence de la République, du point de vue des pouvoirs constitutionnels et du rôle du président (Pereira, 1984 ; Matos, 1983 ; Cruz, 1994 ; Canotilho, Moreira, 1991 ; Lucena, 1996 ; Freire, Pinto, 2005 ; Rapaz, 2013).

On remarque que les fonctions présidentielles sont étudiées sans que les pratiques discursives soient prises en compte. L'actualité et l'adéquation au contexte portugais de l'assertion de Bacot (2007, p. 104), «la science politique parle peu de discours, de langages, et a fortiori, de mots», est manifeste.

Il y a évidemment des références aux discours présidentiels, mais pour mettre en lumière surtout leurs effets politiques : «Le 25 avril, [le président Eanes] fait un discours qui est un avertissement» (Matos, 1983, p. 252) ou "L'énoncé de Delgado [candidat à l'élection présidentielle de 1958, durant la dictature], en se référant à Salazar, a représenté la crise la plus menaçante pour le régime» (Freire, Pinto, 2005, p. 32).

En tout cas, l'objet de recherche n'est pas la construction verbale du politique mais les organisations, les institutions, les relations entre les différents organismes gouvernementaux. Les chercheurs ont recours aux discours en tant qu'outils, c'est-à-dire linguistiquement et discursivement neutres et donc «négligeables» en tant que tels : «Ce qui est dit ou écrit en politique est en quelque sorte considéré en tant que tel, sans intérêt particulier pour la façon dont cela est dit ou écrit »(Bacot, 2007, p 105). La Constitution portugaise est le discours de référence; et il s'agit plutôt de développer une compréhension plus fine de l'institution présidentielle et de ses modes de fonctionnement que d'étudier la façon dont les présidents construisent leurs discours et en quoi cela est politiquement important.

Les études communicationnelles, la deuxième perspective s'intéressant aux discours présidentiels, ont pour objectif premier d'analyser les médias et les reconfigurations du discours politique, en particulier les discours de campagnes électorales, mais aussi les débats et d'autres événements politiques télévisuels ${ }^{6}$. L'objet des sciences de la communication est plus vaste, donc, mais les discours ne sont plus des éléments «négligeables»: "Comprendre et étudier le langage en politique est un facteur de pouvoir qui devrait être un défi pour les chercheurs du «langage de la politique », comme le prônait Lasswell (1949, p. 17)» (Espírito Santo, Figueiras, 2010, p. 83). Toutefois, c’est une démarche d'analyse de contenu qui est choisie (Salgado, 2010 ; Espírito Santo, 2006). L'éloignement face aux sciences du langage est d'ailleurs assumé :

6. Maingueneau (2010, p. 88) y fait référence : «Il s'est produit un brouillage de la frontière entre les études sur les médias et les études sur le discours politique.» 
«Cette étude [...] ne fait pas partie de la matrice théorique linguistique que l'analyse textuelle et discursive peuvent fournir » (Espírito Santo, 2006, p. 85). Bien au contraire, il s'agit d'une «matrice sociologique»: «Nous avons opté pour l'utilisation de l'analyse de contenu [...] compte tenu de son adéquation et de la tradition dans l'analyse [...] de contenus communicationnels» (Espírito Santo, 2006, p. 84). C'est pourquoi l'identification des thématiques abordées en campagne électorale ou dans des discours d'investiture, par exemple, est une des tâches centrales des travaux réalisés.

Un cas spécifique de la médiatisation de la politique en rapport étroit avec les campagnes électorales commence à attirer l'attention des chercheurs portugais : c'est le marketing politique, notamment le phénomène très actuel de spin doctoring, qui réorganise la communication politique en général, celle des présidents notamment. Les recherches rendent compte de ce tournant dans la communication entre les politiques et les médias sans pour autant traiter des questions discursives (Salgado, 2012 ; Ribeiro, 2015).

Finalement, les sciences du langage, qui constituent le troisième versant de ces études sur les discours présidentiels, ont plus récemment intégré cet objet. Les analystes du discours se sont intéressés aux discours parlementaires ou alors aux liens entre les discours politiques et les médias, à côté de travaux sur des discours de campagnes électorales ou de commémoration d'événements nationaux (Marques, 2014; Pinto, 2012 ; Teletin, 2013). Le discours politique pris dans sa matérialité est l'objet de recherche : il n'est pas un simple instrument méthodologique pour l'étude d'autres réalités sociales.

En paraphrasant Micheli (2010), l'apport spécifique du linguiste versé dans l'analyse des discours à l'analyse des discours présidentiels est sans doute l'assomption de la non transparence des discours (Marques, 2014). L'organisation énonciative des discours est alors un point de départ préférentiel pour ces recherches.

Pour les analystes des discours, les images présidentielles dont parlent aussi les chercheurs en science politique et en communication (Rapaz, 2012 ; Salgado, 2012)7 se construisent dans les discours (c'est la problématique de l'ethos). L'attention qu'ils portent aux discours, au dit et aussi au dire, met en relief des stratégies de construction de la crédibilité du président ${ }^{8}$, qui contribuent à la construction de son rôle politique effectif.

7. «Des campagnes [...] qui servent à construire une image de confiance [du candidat]» (Salgado, 2012, p. 244); "Le président de la République portugaise serait la "coupole”, le "sommet de l'ensemble du système institutionnel” ou le "sommet de [sa] pyramide". Il serait la "banque centrale", la "soupape de sécurité" ou "le fusible” du régime politique portugais. Il serait un “arbitre, [un] policier et [un] pompier”, ou un “pater familias” (Rapaz, 2012, p. 521-522).

8. «Le passé personnel, l'histoire démocratique de chaque locuteur, renforce l'ethos positif que la fonction de président (le garant de la démocratie) lui donne et renforce sa crédibilité » (Marques, 2014, p. 305). 
Les discours présidentiels, entre déterminismes institutionnels et stratégies discursives, sont le lieu de la légitimation, de la crédibilisation et du pouvoir des présidents.

Notre démarche pluridisciplinaire a mis en relief des approches scientifiques fort différentes des discours présidentiels; en reprenant les mots de Charaudeau (2010), on a affaire à une « rencontre de disciplines». De prime abord, on dirait que chaque discipline ne s'intéresse guère à ce que les autres disciplines peuvent lui apporter sur ce sujet. Mais en réalité, ces recherches sur les présidents de la République portugaise et leurs discours s'avèrent moins disparates qu'on ne l'aurait pensé. Ce sont, certes, des apports diversifiés, ancrés dans des cadres théoriques et méthodologiques isolés, mais dont les recherches et résultats se recoupent parfois et se complètent, ce qui n'est pas sans importance.

\section{Références}

BACOT Paul, 2007, «Philippe Braud, Sociologie politique», Mots. Les langages du politique, no 84, Politiquement sportif, p. 104-109.

CAnotilho José Joaquim Gomes, Moreira Vital, 1991, Os poderes do Presidente da República, Coimbra, Coimbra Editora.

CAVAco SILVA Anibal, 2007-2015, Roteiros, vol. I-IX, Lisbonne, Imprensa Nacional-Casa da Moeda.

Charaudeau Patrick, 2010, «Pour une interdisciplinarité "focalisée" dans les sciences humaines et sociales», Questions de communication, nº 17, p.195-222.

CRUz Manuel Braga (da), 1994, "O Presidente da República na génese e evolução do sistema de Governo Português", Análise Social. Revista do Instituto de Ciências Sociais da Universidade de Lisboa, vol.XXIX, nº 1-2 [n0125-126], p. 237-265.

Espírito SAnto Paula (do), 2006, «A mensagem política na campanha das eleições presidenciais. Análise de conteúdo dos slogans entre 1976 e 2006 », Comunicação \& Cultura, no 2, p. 83-101.

- 2007, «Comunicação e política nos discursos presidenciais de tomada de posse, 1976-2006 », Revista de estudos em comunicação, nº 2.

Espírito Santo Paula (do), Figueiras Rita, 2010, «Comunicação eleitoral», J.C. Correia, G. Ferreira, P. Espírito Santo éd., Conceitos de comunicação política, Covilhã, LabCom Books, p. 77-89.

Freire André, Pinto Antonio Costa, 2005, O Poder dos presidentes. A República Portuguesa em debate, Lisbonne, Campo da Comunicação.

LASSWELL Harold, 1979 [1949], A Linguagem da Política, Brasília, Editora Universidade de Brasília.

LUCENA Manuel (de), 1996, "Semipresidencialismo. Teoria geral e práticas portuguesas (1) ", Análise Social. Revista do Instituto de Ciências Sociais da Universidade de Lisboa, vol. XXXI, nº 4 [n 138], p. 831-894.

Maingueneau Dominique, 2010, «Le discours politique et son environnement », Mots. 
Les langages du politique, n94, "Trente ans d'étude des langages du politique (1980-2010)», p. 85-90.

MARQUeS Maria-Aldina, 2014, «Palavra de Presidente. Construção da autoridade nos discursos de Abril », Forma breve, n011, Universidade de Aveiro, p. 297-311.

MAtos Luis Salagado (de), 1983, "Significado e consequências da eleição do presidente por sufrágio universal. 0 caso português", Análise Social. Revista do Instituto de Ciências Sociais da Universidade de Lisboa, vol. XIX, nº 2 [nº 76], p. 235-260.

MICHELI Raphaël, 2010, «Les usages du pathos dans les débats parlementaires français sur l'abolition de la peine de mort. Une approche argumentative», communication au colloque international, Faire parler le parlement. Méthodes et enjeux de l'analyse des débats en assemblées politiques, organisé par l'Association française de science politique, Paris, 13-14 octobre 2010, 〈http://www.afsp.msh-paris.fr/activite/groupe/grpp/grpp131010textes/s6micheli.pdf〉 (consulté le 3 juin 2016).

Pereira André Gonçalves, 1984, O semipresidencialismo em Portugal, Lisbonne, Ática.

Pınto Antonio Costa éd., 2001, Os Presidentes da República Portuguesa, Lisbonne, Círculo de leitores.

PInTo Maria Alexandra Guedes, 2012, «Marcas de dialogismo e polifonia nos manifestos políticos presidenciais de 2011 », Estudos Linguísticos / Linguistic Studies, n 8 , p. $195-212$.

RAPAz Paulo José Canelas, 2008, «Le président de la République portugaise : une présidence "neutre" ?», communication au VIIe Congrès français de droit constitutionnel organisé par l'Association française de droit constitutionnel, Cinquantième anniversaire de la constitution de 1958, Paris, 25-27 septembre 2008, <http:// www.droitconstitutionnel.org/congresParis/comC6/CanelasTXT.pdf> (consulté le 3 juin 2016).

- 2012, Le président de la République portugaise. La construction de la figure présidentielle portugaise depuis 1986, Thèse de Doctorat, sciences politiques, Université Paris 2-Assas, 〈http://www.theses.fr/2012PA020075〉 (consulté le 3 juin 2016).

- 2013, «Le président de la République et le régime politique portugais. Entretiens», Jus Politicum, no 10.

Ribeiro Vasco, 2015. «O spin doctoring em Portugal. Perspectivas de governantes, jornalistas e assessores de comunicação que operam na Assembleia da República ». Observatorio (OBS*) Journal, vol. 9, n², p. 225-256.

SAlgado Susana, 2010, Os candidatos presidenciais. Construção de imagens e discursos nos media, Coimbra, Minerva.

- 2012, «Campanhas eleitorais e cobertura mediática. Abordagens teóricas e contributos para a compreensão das interações entre política e media », Revista Brasileira de Ciência Política, nº 9, p. 229-253.

SAmPAIO Jorge, 1997-2007, Portugueses, vol. I-IX, Lisbonne, Imprensa Nacional-Casa da Moeda.

SOARES Mário, 1986-1996, Intervenções, vol. I-X, Lisbonne, Imprensa Nacional.

SousA Marcelo Rebelo (de), 1998, Ciência política. Conteúdos e métodos, Lisboa, Lex.

TELETIN Andreea, 2013, «Les vœux présidentiels au Portugal, en France et en Roumanie, 
MARia-Aldina MARques

et la crise internationale. Les enjeux des formes d'adresse et des procédés d'atténuation/intensification », Mots. Les langages du politique, nº101, "Le discours politique portugais », p. 31-46.

\section{Sitographie}

Présidence de la République portugaise 〈http://www.presidencia.pt` (consulté le 3 juin 2016). 